\title{
Medicina interna, paziente complesso, evidence based medicine e le non evidenze
}

\author{
Internal medicine, complexity, evidence based medicine, \\ almost "without evidences"
}

\section{Roberto Nardi ${ }^{a, *}$, Tatiana Fabbri ${ }^{a}$, Gelorma Belmonte ${ }^{a}$, Paolo Leandri ${ }^{a}$, Magda Mazzetti a , Angelo Pasquale a , Massimo Reta ${ }^{a}$, Claudia Rizzi ${ }^{a}$, Giovanni Scanelli ${ }^{b}$, Ido lori ${ }^{c}$, Gualberto Gussoni ${ }^{d}$, Claudio Pedace ${ }^{e}$, Giovanni Mathieu ${ }^{f}$, Antonino Mazzone ${ }^{g}$}

\author{
"Medicina Interna, Ospedale "Maggiore", Azienda USL di Bologna \\ ${ }^{\mathrm{b}}$ Medicina Interna, Azienda Ospedaliero-Universitaria di Ferrara \\ "Medicina Interna, Azienda Ospedaliera di Reggio Emilia "Arcispedale S.ta Maria Nuova"; Direttore Centro Studi FADOI, \\ Past President FADOI \\ ${ }^{\mathrm{d}}$ Responsabile Scientifico Centro Studi FADOI \\ e Direttore Distretto Sanitario di Arezzo; Segretario Centro Studi FADOI \\ ${ }^{f}$ Medicina Interna, Ospedale di Pinerolo (TO), ASL TO 3; Past President FADOI \\ ${ }^{\mathrm{g}}$ Medicina Interna, Ospedale di Legnano (MI); Presidente Nazionale FADOI
}

Ricevuto il 29 aprile 2009; accettato il 10 maggio 2009

disponibile online il 23 settembre 2009

\section{KEYWORDS \\ Evidence based \\ medicine; \\ Internal medicine.}

\begin{abstract}
Summary
Background: Internal medicine has been defined as the specialty of the adult medical complex patients. Complexity science suggests that illness (and health) results from complex, dynamic, and unique interactions between different components of the overall system. In a patient, complexity involves the intricate entanglement of two or more systems (e.g.; body-diseases, family, socioeconomic status, therapies).

Aim of the study: To evaluate the real applicability of Evidence Based Medicne (EBM) in clinical Departments of Internal Medicine and its critical perspectives.

Discussion: Habitually the internist takes decisions in these situations: a) certainty (the ideal decision is adopted and the corresponding strategy follows), b) risk (the more suitable alternative selected can be the determination of the probable value or mathematical hope) and c) uncertainty, in which decisions linked to triple agents: beliefs and personal values of the doctors (I) for their patients (II) in the society (III). In the medical decisions there are often different
\end{abstract}

\footnotetext{
* Corrispondenza: Medicina Interna, Ospedale Maggiore, l.go B. Nigrisoli 2 - 40100 Bologna.

E-mail: r.nardi@ausl.bo.it (R. Nardi).
} 
factors that go beyond the field of technical and scientific knowledge (family, social, economic problems, etc.) and demanding an ethical analysis of the decision.

Conclusions: The "evidence-based medicine", as other models of care, has - in itself - some limitations. "No evidence in medicine" matters that the postulates of the EBM are not always applicable to the real patients of Internal Medicine wards, mostly elderly, frail, complex, with comorbidities and polipharmacy, often with cognitive dysfunction and limitation of autonomy, with psycho-emotional, social and economic problems. The interacting effects of overall involved diseases/factors and their management require more complex and individualised care than simply the sum of separate guideline components. Further innovation is required to resolve the need to enhance integration of evidence with our patients' values at the "bedside and/or clinic" management.

(c) 2009 Elsevier Srl. All rights reserved.

\section{Introduzione}

Secondo l'originale definizione di David L. Sackett, la "Evidence Based Medicine" (EBM) costituisce un approccio alla pratica clinica in cui le decisioni risultano dall'integrazione tra l'esperienza del medico e l'utilizzo coscienzioso, esplicito e giudizioso delle migliori evidenze scientifiche disponibili, mediate dalle preferenze del paziente [1]. I "buoni dottori" riescono a impiegare l'esperienza clinica e le "prove" della letteratura medica qualificata, ma nessuna di queste componenti può essere sufficiente. La crisi del modello biomedico della malattia, l'eccessivo impiego delle valutazioni probabilistiche, l'enfasi sugli studi clinici randomizzati (effettuati, in realtà, su pazienti "ideali") e, da questi, sulle "linee guida", l'esasperata ricerca di soluzioni positivistiche per la definizione diagnostica (fino a confondere i mezzi con il fine, a causa di una "mentalità tecnologica" dei professionisti, di per sé utile, ma certamente meritevole di un ridimensionamento nell'ambito del ragionamento clinico), il ricorso alle terapie sintomatiche impostate prima della diagnosi, i rischi di un impiego non attento e responsabile delle indagini genetiche costituiscono importanti elementi di riflessione per il medico, oggi, per porsi in una posizione non tanto "contro il metodo, ma soltanto contro i suoi eccessi" [2].

Scopo della presente rassegna è valutare la reale applicabilità della EBM nei reparti ospedalieri di Medicina Interna, considerandone criticità e prospettive.

\section{Paziente complesso e decisioni mediche}

Edgar Morin definisce "complesso" ciò che è caratterizzato da una grande quantità di attori/elementi/componenti in causa e di interazioni fra questi, con il rischio conseguente [3]. Vi è complessità quando le differenti componenti che costituiscono un tutto sono inseparabili e quando vi è un tessuto interdipendente e interattivo fra le parti e il tutto, e il tutto e le parti. La complessità richiede una strategia, che è l" "arte di muoversi nell'incertezza". Complessità significa impossibilità, da parte della scienza, di ridurre in termini lineari non tanto la realtà oggetto di studio, bensì i modi diversi in cui la realtà viene studiata. Il pensiero complesso aspira alla conoscenza multidimensionale e multidisciplinare, ma è consapevole dell'impossibilità della conoscenza completa. Il pensiero complesso è animato da una permanente aspirazione a un sapere non parcellizato, non settoriale, non riduttivo, orientato a sviluppare l'attitudine a interconnettere le conoscenze, a contestualizzare la complessità nel mondo reale: esso richiede una visione competente nell'assistenza, basata su un equo giudizio circa le possibili interazioni tra i diversi componenti in causa $[4,5]$.

\section{Quale definizione di complessità?}

Non esiste una definizione univoca di complessità: in Medicina Interna studiare la complessità significa comprendere il bisogno di ricomposizione, di riaggregazione delle conoscenze, superare la frammentarietà [6]. La complessità rende la Medicina una scienza probabilistica, con un elevato rischio di errore, per l'incertezza che permea le decisioni mediche, che pur devono essere adottate in un tempo limitato e in un contesto di conoscenza non sempre definito.

Secondo la definizione della Facoltà di Sociologia dell'Università di Vienna, la complessità è l'embricarsi delle influenze di due o più sistemi nello stesso individuo (per esempio, malattie della sfera fisico-psichica, contesto sociale ed economico, farmaci). La definizione di "paziente complesso", adottata dall'Agency for Healthcare Research and Quality (AHRQ), si riferisce a una persona affetta da due o più malattie croniche, in cui ciascuna delle condizioni morbose presenti è in grado d'influenzare l'esito delle cure delle altre coesistenti, attraverso varie modalità: la limitazione della speranza di vita, l'aumentata morbilità intercorrente, le interazioni tra terapie farmacologiche, l'impossibilità del pieno impiego di cure adeguate per controindicazione ecc. [7].

Nell'accezione più completa di complessità, le componenti di tipo biologico, socioeconomico, culturale, comportamentale e ambientale diventano tutte importanti determinanti di salute [8], purtroppo - e paradossalmente considerate, nei criteri di eleggibilità, come potenziali "confondenti" ai fini di un" oggettiva" valutazione dei risultati di un trial clinico.

Complessità delle cure può essere intesa anche come una maggiore quantità di tempo da dedicare al paziente per valutare ed erogare l'assistenza necessaria [9]. In Medicina Interna la gestione di un paziente complesso significa, in funzione delle sue caratteristiche specifiche, possedere competenze peculiari per affrontare le sfide insite nel singolo caso (tabella 1) [10]. 
Tabella 1 Competenze e capacità dell'internista necessarie per la gestione del paziente complesso.

\begin{tabular}{ll}
\hline Caratteristiche dei pazienti internistici & Competenze e sfide per l'internista \\
\hline - Adulti, generalmente anziani o molto anziani & - Valutazione clinica, laboratoristica, strumentale \\
- Con diverse comorbilità & e multidimensionale \\
- Con prevalenti malattie croniche/cronico-riacutizzate & - Inquadramento nel setting assistenziale (attuale e futuro) \\
- In alcuni casi "robusti" & e valutazione della situazione \\
- In alcuni casi "fragili" & - Stratificazione del rischio e selezione degli elementi salienti \\
- A volte disabili, a rischio di perdita dell'autosufficienza & - Definizione delle priorità e gerarchizzazione dei bisogni \\
- A volte instabili, in alcuni casi critici & - Selezione degli obiettivi di cura e della terapia farmacologica \\
- Con limitata speranza di vita & appropriata \\
- In trattamento polifarmacologico & - Valutazione evidence based delle prove di efficacia disponibili \\
- Con frequenti ricoveri & - Decision making in condizioni d'incertezza \\
- In alcuni casi a rischio di dimissione difficile & - Stratificazione prognostica \\
- Che richiedono il giusto tempo di ascolto e di valutazione & - Differenziazione nell'intensità delle cure \\
- Che richiedono l'intervento di diversi specialisti & - Relazione di cura ed empatia con il paziente \\
- Condivisi fra diversi medici nel processo di assistenza & - Relazioni con i famigliari e i caregiver \\
- Che richiedono continuità assistenziale & - Relazioni e interazioni con gli specialisti \\
& - Educazione al self management \\
& - Coordinamento e comunicazione \\
& - Pianificazione e care case management
\end{tabular}

Fonte: modificata da Nardi R, et al. Eur J Intern Med 2007;18(5):359-68.

Tabella 2 Livelli di consapevolezza in funzione delle decisioni del medico internista.

\begin{tabular}{|c|c|c|}
\hline Livello I & Livello II & Livello III \\
\hline $\begin{array}{l}\text { Comporta la capacità di percezione e selezione } \\
\text { delle informazioni e degli spunti salienti } \\
\text { dall'ambiente }\end{array}$ & $\begin{array}{l}\text { Comporta la capacità di } \\
\text { integrazione delle informazioni, che } \\
\text { consente di assumere le decisioni }\end{array}$ & $\begin{array}{l}\text { È il più alto livello di comprensione della } \\
\text { situazione, da parte di un esperto } \\
\text { qualificato e competente, in grado di } \\
\text { valutare gli elementi clinici, } \\
\text { la letteratura scientifica, il contesto }\end{array}$ \\
\hline $\begin{array}{l}\text { Corrisponde al processo di acquisizione delle } \\
\text { informazioni attraverso la storia clinica, } \\
\text { l'anamnesi farmacologica, l'esame } \\
\text { fisico e i test diagnostici }\end{array}$ & & $\begin{array}{l}\text { Corrisponde alla capacità di prevedere, } \\
\text { con grande verosimiglianza, la possibile } \\
\text { evoluzione clinica, oltre le singole } \\
\text { prestazioni professionali e i possibili } \\
\text { risvolti futuri, anche in termini gestionali } \\
\text { e di rischi potenziali, in funzione di una } \\
\text { valutazione dinamica del processo di cura }\end{array}$ \\
\hline \multicolumn{3}{|l|}{ Ai fini della decisione del medico } \\
\hline $\begin{array}{l}\text { Quali sono gli elementi salienti di questo } \\
\text { paziente? }\end{array}$ & $\begin{array}{l}\text { Che cosa sta succedendo } \\
\text { a questo paziente? } \\
\text { Quali sono le priorità? } \\
\text { Che cosa dobbiamo fare? }\end{array}$ & Che cosa è più probabile che accada se...? \\
\hline
\end{tabular}

Fonte: modificata da Singh H, et al. Qual Saf Health Care 2006;15(3):159-64.

La complessità pone all'internista obiettivi non solo di miglioramento della sua competenza (intesa come sapere, saper fare, saper essere), ma anche della sua adattabilità al cambiamento, attraverso nuove conoscenze e con i massimi livelli di consapevolezza in relazione al contesto (situation awareness), ai fini della decisione più adeguata (tabella 2) [11].

La vera sfida consiste nel saper migliorare le proprie capacità, attraverso una valutazione sistemica dei bisogni e della prognosi dei pazienti, andando oltre le singole prestazioni, considerando il processo assistenziale nel suo insieme, filtrando le utili opinioni specialistiche mediante la "regia" dei casi, con un controllo e un feedback sistematico delle interazioni esistenti fra gli attori/elementi in causa e dei rischi possibili, anche in condizioni di incertezza $[12,13]$.

\section{La limitazione dell'applicabilità della EBM non significa che essa non sia utile agli internisti}

La EBM ha una diffusione disomogenea nella pratica medica. Nel 1991 è stato stimato che solamente il 15\% degli interventi medici fosse sostenuto da prove scientifiche 
Tabella 3 L'utilità della EBM nelle diverse fasi del procedimento clinico.

\begin{tabular}{ll}
\hline Fase & Utilità \\
\hline $\begin{array}{l}\text { Riconoscimento di presentazioni } \\
\text { cliniche e formulazione delle } \\
\text { ipotesi diagnostiche }\end{array}$ & Nessuna/scarsa \\
\hline $\begin{array}{l}\text { Varifica/conferma delle ipotesi } \\
\text { (scelta e interpretazione }\end{array}$ & Minima/moderata \\
dei test diagnostici) & \\
\hline $\begin{array}{l}\text { Decisioni terapeutiche (scelta } \\
\text { e valutazione dei trattamenti) }\end{array}$ & Nessuna/scarsa/ \\
\hline
\end{tabular}

consolidate [14]. Secondo dati più recenti, con riferimento ai medici di varie specializzazioni, la percentuale delle decisioni basate sulle regole della EBM varia dall'11 al 70\% [15]. Gli specialisti d'organo e/o di apparato tendono a utilizzare maggiormente la EBM, probabilmente per il più limitato campo d'azione, riferito a pazienti con problemi "omogenei", ben rappresentati nei grandi trial clinici, al contrario di quelli - più complessi - trattati dagli internisti $[16,17]$.

Nonostante tali valutazioni, nell'ultimo decennio la EBM ha determinato, in diverse generazioni di medici, un cambiamento culturale "radicale", impossibile da ignorare, in tutti gli ambiti di assistenza sanitaria, pratica professionale, formazione, pianificazione della politica sanitaria e della ricerca, comunicazione con i pazienti e i cittadini [18].

\section{II giudizio clinico in un contesto complesso: l'epidemiologia dell'incertezza}

Il medico, schematicamente, si trova a operare in tre tipi di contesto.

- Condizioni di certezza: gli elementi in causa sono ben noti e inquadrabili, la malattia è una sola, la terapia consolidata e conosciuta. È la situazione ideale, in cui la strategia di gestione è semplice.

- Condizioni di rischio: la strategia alternativa migliore possibile viene selezionata tra quelle note, in base a criteri probabilistici e alle prove di efficacia, se disponibili.
- Condizioni di incertezza: possono riguardare variabili biologiche, cliniche, psicosociali, economiche ecc. [19].

Le decisioni si fondano sul "giudizio clinico" del medico, ponderato in base a una valutazione fisiopatologica, alle prove di efficacia (se disponibili), alle priorità emergenti e alle propensioni del paziente, in funzione del contesto sociale e normativo e a imprescindibili considerazioni etiche: tale situazione è di gran lunga quella in cui più frequentemente si trova a operare il medico internista. Considerando le diverse fasi del procedimento clinico, in ambito diagnostico il contributo della EBM è, nella maggioranza dei casi, limitato o di utilità non sempre univoca (tabella 3 ).

In ambito terapeutico, considerando l'efficacia e/o la potenziale nocività di diversi tipi di trattamento (in base alle prove disponibili), è stato documentato che solamente il $38 \%$ degli interventi può essere ritenuto "utile o potenzialmente utile". "Prove chiare" - sia di segno positivo (utili) sia di segno negativo (verosimilmente inefficaci o dannose) - possono essere fornite solamente nel $19 \%$ dei casi (tabella 4) [20]. Questi dati confermano le condizioni d'incertezza del mondo reale, in cui l'internista è chiamato a operare.

\section{Trial clinici randomizzati e mondo reale}

L'applicabilità di uno studio clinico randomizzato (RCT), ovvero la sua validità esterna (generalizability o effectiveness), è il grado con cui i risultati della ricerca possono essere applicati al paziente individuale nel contesto reale. Essa è influenzata principalmente dai criteri di selezione/ esclusione dei pazienti dello studio, dal setting assistenziale, dagli aspetti organizzativi, dalle tecnologie, dalla clinical competence, dalla capacità dei pazienti di aderire alle cure, ma anche dalla significatività e rilevanza clinica dei risultati ottenuti. Le prospettive del ricercatore-metodologo e del clinico pratico sono differenti (tabella 5).

\section{Medicina basata sulle evidenze e Medicina incentrata sul paziente}

Una Medicina etica, oggi enfaticamente definita come "incentrata sul paziente", richiede al medico di occuparsi di ciascun paziente come persona [21]. Nell'assistenza al

Tabella 4 Categorizzazione degli effetti dei trattamenti.

\begin{tabular}{lll}
\hline Effetti del trattamento & Descrizione & (\%) \\
\hline Utili & $\begin{array}{l}\text { Interventi la cui efficacia è stata ben documentata da prove chiare, con } \\
\text { benefici elevati e minimo rischio di danno }\end{array}$ \\
\hline Potenzialmente utili & Interventi la cui efficacia è meno evidente & 15 \\
\hline $\begin{array}{l}\text { Da valutare nelle specifiche } \\
\text { circostanze }\end{array}$ & $\begin{array}{l}\text { Interventi che i clinici, insieme ai pazienti, dovrebbero valutare, soppesando } \\
\text { i benefici e i rischi in riferimento al contesto specifico e in funzione delle } \\
\text { priorità }\end{array}$ \\
\hline Probabilmente non utili & Interventi la cui mancata efficacia non è del tutto stabilita & 8 \\
\hline $\begin{array}{l}\text { Verosimilmente inefficaci } \\
\text { o dannosi }\end{array}$ & Interventi la cui inefficacia e nocività sono documentate da prove chiare
\end{tabular}

Fonte: Liberati A. Centro Cochrane Italiano, XIII Riunione Annuale Network Cochrane Italiano. Workshop. Napoli, 4 novembre 2008. 
Tabella 5 Prospettive del ricercatore-metodologo e del clinico pratico riguardo agli studi clinici.

\begin{tabular}{ll}
\hline $\begin{array}{l}\text { Prospettiva del ricercatore } \\
\text { e del metodologo }\end{array}$ & $\begin{array}{l}\text { Prospettiva del } \\
\text { medico pratico }\end{array}$ \\
\hline - Approccio EBM & - Approccio \\
& fisiopatologico e clinico \\
- Popolazione degli RCT & - Popolazione reale \\
- Qualità degli RCT & - Applicabilità degli RCT \\
- Certezze nella EBM & - Zone grigie della EBM \\
- Significatività statistica & - Rilevanza clinica \\
- "Explanatory" trial & - "Pragmatic" trial \\
- Efficacy & - Effectiveness \\
- Importanza dell'efficacia & - Importanza della \\
comparativa & farmacovigilanza \\
- Qualità delle linee guida & - Applicabilità delle \\
& linee guida \\
\hline
\end{tabular}

paziente, l'internista dovrebbe astrarsi da assunti preconcetti. È umano, naturale ed etico che la propensione del clinico, a fianco e al letto del paziente, sia prevalentemente orientata a un'interazione diretta con l'ammalato, per considerarne specificità e bisogni, che, a volte, sono anche inespressi o non espliciti (tabella 6).

\section{La popolazione rappresentata nei trial randomizzati e controllati: criteri di eleggibilità/esclusione}

Uno dei problemi più critici, inerente la generalizzazione dei risultati di un RCT, riguarda i criteri di eleggibilità/esclusione da uno studio. Il profilo del "paziente tipo" ammissibile per uno studio clinico corrisponde, in generale, a un soggetto di sesso maschile, giovane adulto, affetto da una sola malattia, più spesso acuta o subacuta, senza compromissioni funzionali, con un'ottima compliance, seguito con un follow-up di durata limitata, con valutazione della effectiveness piuttosto che della safety/tolerability, controllato con placebo, in alcuni casi con endpoint surrogati [22].

Al contrario, il "mondo reale" dei pazienti ricoverati in ospedale è più spesso costituito da donne, affette da malattie croniche, degenerative, progressive, con associate frequenti sindromi geriatriche (cadute: 31\%/anno; delirium, demenza: prevalenza del 10\%; incontinenza urinaria: prevalenza del $15-30 \%$; dolore cronico: prevalenza del 25-50\%; depressione: presente nel $15 \%$ dei pazienti nel territorio) e numerose altre comorbilità, perdita dell'autonomia, in trattamento polifarmacologico. Emergono, di conseguenza due osservazioni:

- L'impiego selettivo di stringenti criteri di eleggibilità pone evidenti problemi di bias di selezione, comportando più

Tabella 6 Differenze sostanziali fra EBM e Medicina incentrata sul paziente.

\begin{tabular}{|c|c|}
\hline Medicina basata sulle evidenze & Medicina incentrata sul paziente \\
\hline $\begin{array}{l}\text { - Disease-centered (best care of the disease): } \\
\text { viene valutata la migliore evidenza disponibile } \\
\text { in riferimento alla malattia in causa }\end{array}$ & $\begin{array}{l}\text { - Patient centered (best care of illness): in base a bisogni, ansie, } \\
\text { paure e aspettative del paziente e alla valutazione del rapporto } \\
\text { beneficio/rischio }\end{array}$ \\
\hline $\begin{array}{l}\text { - Approccio biomedico: integrazione delle evidenze } \\
\text { scientifiche con l'esperienza del medico e le } \\
\text { aspettative del paziente }\end{array}$ & $\begin{array}{l}\text { - Approccio umanistico, biopsicologico, interattivo ed empatico } \\
\text { con il paziente, con applicazione delle evidenze scientifiche } \\
\text { (quando e se disponibili) in base a una giusta valutazione dei } \\
\text { problemi prioritari, anche inespressi }\end{array}$ \\
\hline $\begin{array}{l}\text { - Base sperimentale forte, prevalentemente } \\
\text { mediante studi clinici randomizzati effettuati } \\
\text { in gruppi di pazienti omogenei }\end{array}$ & $\begin{array}{l}\text { - Base pragmatica forte, prevalentemente fondata su studi } \\
\text { osservazionali in pazienti appartenenti al mondo reale }\end{array}$ \\
\hline $\begin{array}{l}\text { - L'analisi post-hoc di sottogruppi di pazienti } \\
\text { tende ad "aggiustare il tiro" su categorie } \\
\text { specifiche di pazienti }\end{array}$ & $\begin{array}{l}\text { - L'individualità del singolo paziente costituisce la base di } \\
\text { riferimento per il medico e le sue scelte }\end{array}$ \\
\hline $\begin{array}{l}\text { - Rigidi criteri di ammissione/esclusione dei } \\
\text { pazienti negli studi clinici }\end{array}$ & $\begin{array}{l}\text { - Comorbilità e complessità dei pazienti del mondo reale come } \\
\text { elemento imprescindibile di contesto }\end{array}$ \\
\hline $\begin{array}{l}\text { - Selezione metodologicamente corretta di un } \\
\text { campione, conseguente inferenza e } \\
\text { valutazione della significatività statistica }\end{array}$ & $\begin{array}{l}\text { - Esperienza, capacità discriminativa degli elementi clinici } \\
\text { salienti, rilevanza clinica più che statistica }\end{array}$ \\
\hline $\begin{array}{l}\text { - Attore principale: il paziente non "interagisce" } \\
\text { mai con l'epidemiologo statistico metodologo }\end{array}$ & $\begin{array}{l}\text { - Attore principale: il paziente ha al fianco del suo letto il clinico, } \\
\text { che deve decidere in un prevalente contesto di complessità, } \\
\text { in una relazione di cura orientata all'ascolto dei bisogni espressi } \\
\text { e inespressi del paziente }\end{array}$ \\
\hline $\begin{array}{l}\text { - Prevale il giudizio sulla regolarità della "metodologia } \\
\text { della ricerca": si valuta se vengono rispettati } \\
\text { i consolidati obiettivi della "ricerca } \\
\text { metodologicamente corretta" } \\
\text { - In ultima analisi: prevale l'attenzione alla validità } \\
\text { interna e metodologica dello studio }\end{array}$ & $\begin{array}{l}\text { - Prevale il "giudizio clinico": la capacità di saper gerarchizzare } \\
\text { i bisogni del paziente e selezionare le priorità, in base a un } \\
\text { ragionamento sia fisiopatologico sia di valutazione } \\
\text { multidimensionale, contestualizzato al setting assistenziale } \\
\text { - In ultima analisi: viene richiesta la possibilità di applicare } \\
\text { ai pazienti "bed side" i risultati degli studi clinici, ovvero la } \\
\text { validità esterna e l'applicabilità dei risultati dello studio }\end{array}$ \\
\hline
\end{tabular}


Tabella 7 Criteri di ammissibilità ed esclusione (giustificata/ingiustificata) da un RCT.

\begin{tabular}{lll}
\hline Ammissibilità dell'esclusione da un RCT & $\begin{array}{l}\text { Ingiustificata esclusione } \\
\text { da un RCT }\end{array}$ & $\begin{array}{l}\text { Esclusione da un RCT potenzialmente } \\
\text { giustificata }\end{array}$ \\
\hline - Impossibilità di ottenere un consenso informato & - Età & - Il soggetto potrebbe non aderire al \\
- Possibilità che il trattamento sperimentale & - Sesso & trattamento \\
oppure il placebo siano nocivi al soggetto & - Condizioni correlate & - Il paziente potrebbe non essere in grado \\
arruolato & al genere (mestruazioni, & di completare il follow-up \\
- Possibilità che il trattamento non sia efficace & gravidanza, allattamento ecc.) & - La speranza di vita del paziente non \\
in quanto il soggetto arruolato: & - Apparttenza a particolari & consente la sua eleggibilità allo studio \\
a) ha scarse probabilità di ottenere l'esito di & gruppi etnici o religiosi & \\
interesse; & - Grado di istruzione & \\
b) non è a rischio di sviluppare l'esito di & - Stato socioeconomico & \\
interesse; & - Stato cognitivo & \\
c) ha un tipo di patologia che non risponde & - Abilità o disabilità fisica & \\
al trattamento & - Presenza di patologie croniche & \\
- Possibilità che gli effetti dell'intervento & e comorbilità di vario tipo & \\
siano difficili da interpretare in quanto & & \\
il soggetto: & \\
a) è in trattamento con altri farmaci che & \\
possono confondere il reale effetto del & \\
trattamento sperimentale; & \\
b) ha patologie simili all'esito sotto & \\
osservazione, che rendono difficile & \\
l'interpretazione dell'effetto del & \\
trattamento sperimentale & \\
\hline
\end{tabular}

Fonte: Van Spall HG, et al. JAMA 2007;297(11):1233-40.

facili "benefici" di risultato, dato che questi studi, effettuati su popolazioni selezionate, producono risultati generalmente più solidi;

- se gli studi preregistrativi sui nuovi farmaci vengono disegnati su popolazioni altamente selezionate, esiste il rischio (eticamente molto opinabile) di escludere dagli RCT pazienti che hanno un'elevata probabilità di ricevere il trattamento nella pratica clinica.

Fin dal 1994 l'EMEA aveva espresso il principio generale secondo cui " $i$ farmaci devono essere studiati in soggetti di tutte le fasce di età, inclusi gli anziani" e "i pazienti che partecipano agli studi clinici devono essere rappresentativi della popolazione che poi sarà trattata con il farmaco allo studio" [23]. In un lavoro recente, che ha valutato i motivi di esclusione dai trial clinici pubblicati su riviste di alto impatto in Medicina Interna in base a criteri espliciti (tabella 7), è stato dimostrato che l'età rappresenta la principale motivazione per l'esclusione dagli RCT nel $72,1 \%$ dei casi [24].

I soggetti pediatrici sono stati esclusi nel 60,1\% degli RCT, mentre gli anziani di età superiore a 65 anni nel 38,5\% dei casi. Il sesso femminile o condizioni a esso correlate hanno rappresentato un criterio specifico di esclusione addirittura nel $47,0 \%$ degli RCT (il sesso maschile ha rappresentato uno specifico criterio di esclusione solo nel 7,8\% degli studi). La presenza di patologie concomitanti complessivamente ha costituito un criterio di esclusione nell' $81,3 \%$ degli RCT e in molti casi $(30,9 \%)$ non sono stati chiariti né il tipo di patologia né le ragioni.

L'applicazione dei criteri sopra descritti per l'ammissibilità o ingiustificata esclusione dei soggetti indica che l'84,1\% degli RCT conteneva almeno un criterio ingiustificato di esclusione.
Su 2.709 criteri analizzati solo il 47,2\% trova ampia giustificazione nel contesto dell'RCT valutato, il 15,9\% risulta potenzialmente giustificabile e il $37,1 \%$ appare ingiustificato. Ancora una volta l'età $(78,4 \%)$, il sesso femminile $(52,6 \%)$ e la presenza di patologie concomitanti $(64,8 \%)$ sono le categorie per le quali l'esclusione risulta maggiormente ingiustificata. L'analisi multivariata ha rilevato un'associazione indipendente tra il numero dei criteri di esclusione e $i$ trial d'intervento farmacologico $(\mathrm{RR}=1,35 ;$ IC $95 \%=1,11-1,65)$ e multicentrici $(R R=1,26 ;$ IC $95 \%=1,06-1,52)$. Gli RCT sponsorizzati dalle industrie farmaceutiche tendono a escludere maggiormente soggetti anziani con diverse comorbilità e in trattamento concomitante con altri farmaci. Sia l'esclusione per patologie concomitanti sia quella associata ad altri trattamenti farmacologici è risultata ingiustificata.

\section{Trial clinici randomizzati, studi osservazionali, "explanatory/pragmatic" trial e gerachia dei modelli di ricerca}

In una Medicina incentrata sui pazienti del "mondo reale", la migliore evidenza non può derivare solamente dagli RCT ma, probabilmente, anche da studi osservazionali ben condotti $[25,26]$, che i medici dovranno saper interpretare nella loro pratica clinica, proprio per distinguere le ricerche di buona qualità da quelle mediocri [17]. I risultati dello studio di Van Spall [24] confermano la necessità di poter disporre, oltre che di studi finalizzati a dimostrare l'efficacia degli interventi, di studi pragmatici in grado di documentarne l'applicabilità [27]. Sia gli "explanatory" sia i "pragmaticpractical" trial hanno un ruolo importante nella valutazione 
Tabella 8 Caratteristiche salienti degli "explanatory/pragmatic-practical" trial.

\begin{tabular}{ll}
\hline "Explanatory" trial & "Pragmatic-practical" trial \\
\hline - Di impiego comune & - Meno utilizzati \\
- L'obiettivo è dimostrare l'efficacia, in condizioni ideali & - L'obiettivo è verificare i benefici di un intervento nel "mondo \\
- La numerosità del campione può essere anche limitata & reale" \\
- Sono condotti in un setting sperimentale & - La numerosità del campione dovrebbe essere consona alla \\
- Sono condotti su campioni omogenei & rilevanza clinica da evidenziare \\
- Sono controllati vs placebo & - Sono condotti nel setting "routinario" del mondo reale \\
- Sono randomizzati, controllati e condotti in cieco & - Sono condotti su campioni eterogenei \\
- Hanno un'elevata validità interna & - Sono controllati vs "usual care" o vs "best evidence care" \\
- Sono condotti prevalentemente per pazienti acuti & - Sono randomizzati, controllati, spesso non in cieco \\
- Il follow-up è generalmente breve & - Sono rappresentativi della popolazione usuale di pazienti \\
- Sono poco generalizzabili & - Sono rivolti prevalentemente a malattie croniche \\
& - Il follow-up è più prolungato \\
& - Hanno un'elevata generalizzabilità \\
\hline
\end{tabular}

Fonte: modificata da Macpherson H. Complement Ther Med 2004;12(2-3):136-40.

dell'efficacia degli interventi di assistenza sanitaria, in quanto rispondono a quesiti diversi della ricerca. Gli studi pragmatici sono utili per valutare l'efficacia di un trattamento rispetto a standard terapeutici consolidati nella pratica (usual care), normalmente accettati (tabella 8) [28].

Per poter perseguire l'obiettivo di disporre di studi clinici "pragmatici", è necessario che il loro disegno sia orientato ad alcuni elementi sostanziali, sintetizzati nel box 1 [29].

Una prescrizione "ritagliata" sul paziente individuale costituisce un obiettivo terapeutico assolutamente imprescindibile che, però, contrasta con i postulati della EBM, per l'impossibilità di confrontare $i$ benefici di diverse terapie in presenza di prognosi differenti (confondimento da indicazione). Le revisioni sistematiche dovranno, nel prossimo futuro, integrare i dati ottenuti dagli RCT e dagli studi osservazionali al fine di valutare efficacemente sia i benefici sia i danni delle terapie [30,31]. L'importanza degli studi osservazionali è documentata dal fatto che i risultati di questi studi, se ben disegnati, non sovrastimano l'entità degli effetti del trattamento rispetto agli RCT [32].

\section{Significatività statistica e rilevanza clinica}

Tra gli elementi salienti che differenziano il punto di vista dell'epidemiologo-metodologo e quello del clinico sono compresi i concetti di significatività statistica e di rilevanza clinica, che non sempre coincidono: risultati "statisticamente significativi" possono essere clinicamente non importanti e, viceversa, effetti terapeutici "statisticamente non significativi" possono veicolare il messaggio di una terapia clinicamente utile [33].

La significatività statistica è un concetto probabilistico: rappresenta la probabilità di ottenere un falso positivo quando si afferma che esiste un certo effetto. Per convenzione, per sostenere che un effetto è statisticamente significativo, questa probabilità deve essere inferiore al $5 \%(p<0,05)$. La significatività statistica è finalizzata a definire quanto è probabile che la differenza osservata nei gruppi di studio di un RCT sia reale o dovuta al caso. La significatività statistica

\author{
Box 1. \\ Elementi fondanti della valutazione degli studi \\ clinici "pragmatici" \\ CRITERI dI ARRUOLAMENTO (INCLUSIONE/ESCLUSIONE) \\ OBIETTIVI \\ CARATTERIZZAZIONE DEL PAZIENTE \\ StRATEGIE PER AUMENTARE ADESIONE E PARTECIPAZIONE ALLO STUDIO \\ Problematiche ETICHE, IN PARTicolare CONSENSO INFORMATO \\ DURATA \\ VALUTAZIONE DELLA SICUREZZA
}

non può essere automaticamente trasferita nella pratica clinica.

La significatività - o, meglio, la rilevanza - clinica definisce l'importanza della differenza rilevata negli esiti clinici tra i pazienti trattati e non trattati. In sostanza, è necessario chiedersi quali siano le implicazioni pragmatiche dei dati, in particolare nella valutazione di esiti clinicamente importanti (mortalità totale o causa-specifica, eventi non fatali, morbilità, number needed to treat ecc.) e quanto essi siano utili - o clinicamente rilevanti - nella pratica. La dimostrazione di un effetto clinicamente rilevante dovrebbe essere l'obiettivo primario di ogni studio. Esso può essere definito come il minimo beneficio ottenibile, tale da indurre il medico a cambiare strategia nei confronti del paziente, per esempio consigliando un nuovo trattamento o verificando la presenza di un certo fattore di rischio per correggerlo. In altri termini, la differenza minima clinicamente importante (Minimal Clinically Important Difference, MCID) [34,35] fra una terapia sperimentale e la rispettiva terapia di controllo dovrebbe essere sufficiente a giustificare un cambiamento nelle decisioni terapeutiche, tenendo anche conto di possibili eventi avversi, inconvenienti e costi.

La MCID è un concetto chiave sia nel disegno sia nell'interpretazione dei risultati degli RCT. Nel disegno, infatti, la numerosità del campione (sample size) del trial dovrebbe riflettere la MCID che si vuole evidenziare fra il trattamento in sperimentazione e quello di controllo 
Box 2.

Principali limitazioni delle attuali linee guida [42]

SONO ORIENTATE A UNA SPECIFICA PATOLOGIA

SONO BaSATE SU RCT ALTAMENTE SELEZIONATI, RARAMENTE DEDICATI AL PAZIENTE

ANZIANO CON COMORBILITÀ

Non SONO basate SU STUdI CONDOTTI IN PAZIENTI COMPLESSI E "FRAGILI"

Le raccomandazioni sono limitate al paziente "IDEale"

LE RACCOMANDAZIONI NON CONTEMPLANO L'ETEROGENEITÀ DEL PAZIENTE

IN TERMINI DI:

1) COMORBILITÀ

2) POLIFARMACOTERAPIA

3) STATO FUNZIONALE E COGNITIVO

4) STATO SOCIOFAMILIARE

5) SETTING ASSISTENZIALE

(valore delta). Nell'interpretazione, il raggiungimento o no di una MCID è il criterio principale di cui tener conto nella prospettiva di applicare i risultati del trial alle decisioni terapeutiche.

\section{Limiti delle linee guida nel paziente complesso polipatologico}

L'attuale diffusione delle linee guida (LG), basate sulla valutazione e selezione di studi rigorosi, può portare a miglioramenti rilevanti dell'assistenza sanitaria, ma la qualità "evidence based" delle LG è ben lungi dall'essere ottimale, anche quando proposte da Società scientifiche di tutto rispetto. L'American College of Cardiology e l'American Heart Association (AHA/ACC) hanno recentemente documentato che la proporzione delle raccomandazioni sviluppate su livelli più bassi di evidenza o fondate sul parere di esperti, per le quali non sono documentate prove conclusive, è in progressiva crescita [36]. Inoltre, vi è una base insufficiente di evidenze che, in circostanze complesse, l'implementazione delle LG sia efficace. L'entusiasmo per le LG deve essere sempre temperato dalla consapevolezza che possono fallire. È sempre necessario un attento giudizio del medico sul singolo caso, prima di applicare le indicazioni generali delle LG [37,38].

Le LG possono presentare diverse limitazioni (box 2). Sono troppo spesso incentrate sulle singole patologie e non sono mirate al singolo ammalato. I pazienti raramente hanno singole malattie e poche - se non nessuna - LG sono in grado di supportare il medico nel gestire in maniera flessibile i casi complessi e garantire la personalizzazione delle cure. Le LG sono, in alcuni casi, anche troppo complete e minuziose, descrivendo ogni possibile intervento che potrebbe essere appropriato per un paziente con una singola malattia [39], fino al paradosso di non facilitare, specialmente nei pazienti pluripatologici, l'assunzione di decisioni univoche da parte del medico [40]. È stato ben documentato, d'altronde, che una pedissequa adesione alle LG negli anziani può avere effetti secondari rilevanti $[41,42]$. È necessario, pertanto, che il medico sappia estrapolare gli elementi più solidi dalle LG, per evitare errori indesiderati e conferire valore aggiunto all'assistenza del suo paziente [43].

\section{Il coinvolgimento del paziente nell'esplicitazione delle preferenze e delle scelte assistenziali non è sempre semplice}

La EBM richiede al medico di integrare i valori e le preferenze del paziente nelle decisioni cliniche. Coinvolgere gli ammalati nella loro cura è un processo importante, per esempio nella definizione dei "patient-reported outcomes" [33,44], ma complesso e non sempre applicabile. Diversi elementi interferenti (emozioni, disfunzione cognitiva, depressione, stato di coscienza, presenza di più patologie organiche, stato sociale ed economico, solitudine ecc.) rendono difficile il normale coinvolgimento del paziente. Non tutti desiderano essere coinvolti alla stessa maniera nel processo di cura, a volte anche per un mancato adattamento psicodinamico alla malattia e un rifiuto della stessa [45]. Le persone sono portatrici non solo di una malattia, ma di un'intera vita vissuta, con priorità e valori che, nella vita reale, incidono sulle decisioni diagnostiche e terapeutiche [18], nonché sulla prognosi. Per il medico esiste un'intrinseca difficoltà non solo nel valutare efficacemente i desideri del paziente, ma anche nel trasmettere le informazioni necessarie, che nella Medicina delle prove di efficacia sono spesso fonfate su criteri probabilistici, non facilmente comprensibili da parte dei pazienti.

Queste variabili, così come vari altri fattori di tipo demografico e culturale, possono influenzare la qualità e i risultati della relazione di cura con il paziente [46].

Le preferenze individuali del paziente non sono sempre prevedibili, possono cambiare in relazione alla consapevolezza di malattia e allo stato di sofferenza e possono differire dalle indicazioni delle linee guida [47-49]. Vi è una grande scarsità di riferimenti su come i medici possano riuscire a condividere le evidenze della letteratura scientifica con i loro pazienti per le decisioni da intraprendere insieme. Gli ambiti (e le competenze) da sviluppare sono la comprensione del malato (e della famiglia) e delle sue aspettative, la costruzione di una solida alleanza fra équipe curante e paziente, la spiegazione semplice delle conoscenze disponibili integrata dalla formulazione esplicita del parere del medico, il controllo della comprensione di quanto discusso, ai fini della riuscita del piano diagnostico-terapeutico [50]. In alcuni casi, per mediare con la soggettività del paziente e approdare all" alleanza di cura", si può verificare una sorta di "dissonanza cognitiva", ovvero un'incoerenza per la quale ogni medico reagisce, secondo la propria personalità, fino, in alcuni casi, ad "accontentare" il paziente, in un conflitto tra azione professionale suggerita dalla teoria e quella effettivamente praticata [18].

\section{Conclusioni}

La scienza clinica e l'esperienza del medico hanno entrambe un ruolo fondamenentale in Medicina Interna e devono necessariamente essere complementari. Non è possibile sostituire l'una con l'altra, privilegiando le tecniche di calcolo quantitativo e statistico rispetto alla capacità di 
intuizione e di ragionamento clinico [51], senza incorrere in errori di tipo dogmatico e manicheo.

La EBM, come altri modelli di cura, ha - di per sé - alcuni limiti intrinseci. La Medicina basata sulle prove è l'insieme di competenze che consentono la valutazione critica della letteratura. Le prove della EBM sono in molti casi "grigie", non definitive e a volte addirittura contrastanti, ma le decisioni del medico devono essere, sul campo, "bianche o nere". La EBM necessita di un prerequisito: che l'evidenza sia basata sulla Medicina! In altri termini, la EBM è necessaria ma non sufficiente per la pratica clinica [52]. Studi clinici controllati e revisioni sistematiche servono se sono patrimonio dei clinici, che ne divengono protagonisti attivi. Sono indispensabili ulteriori nuove evidenze, impostate in base a studi clinici controllati e/o osservazionali maggiormente calati nella realtà di ogni giorno; altrettanto urgente è promuovere altresi revisioni sistematiche su problemi concreti di diagnosi e terapia.

L'EBM non deve rimanere patrimonio di pochi, ma diventare strumento fruibile da parte di tutti i medici e, soprattutto, applicabile al maggior numero possibile di pazienti [53]. Così come l'intuizione o il razionale fisiopatologico - da soli - non possono essere sempre adeguati per l'esercizio di processi decisionali, anche il saper utilizzare al meglio le prove empiriche non è sempre possibile, sia perché per certi casi non esistono studi clinici sia perché gli studi effettuati sono di qualità scadente o hanno condotto a conclusioni differenti, o perché i trial sono stati effettuati su popolazioni troppo selezionate [54]. Insomma, come afferma Edgar Morin [3], "la conoscenza è una navigazione in un oceano di incertezze attraverso arcipelaghi di certezze".

Questa consapevolezza non ha, però, necessariamente, una connotazione negativa tale da indurre allo scetticismo, né, tantomeno, all'inerzia decisionale. Essa configura l'opportunità di accettare la complessità come ambito d'azione e non come limite, per una conoscenza più pertinente, con l'uso costante della razionalità come imprescindibile barriera contro l'errore. In sostanza, il medico deve essere in grado di estrapolare, dalla complessità del paziente, la strutturazione del quesito prevalente, di individuare la "best available evidence", di possedere la capacità di valutazione critica della validità e trasferibilità delle informazioni (critical appraisal). Nella valutazione dell'efficacia clinica delle scelte d'intervento, l'internista dovrebbe sempre chiedersi se queste funzionano, e producono più effetti positivi che negativi (efficacy), se funzionano nelle circostanze di pratica clinica abituale (effectiveness) e se "ne vale la pena", considerandone gli effetti in relazione alle risorse utilizzate (efficiency) e ai rischi potenziali e/o attuali (risk management).

\section{Conflitto di interesse}

Gli autori dichiarano di essere esenti da conflitto di interessi.

\section{Bibliografia}

[1] Sackett DL, Rosenberg WM, Gray JA, Haynes RB, Richardson WS. Evidence based medicine: what it is and what it isn't. BMJ 1996;312(7023):71-2.

[2] Cagli V. La crisi della diagnosi. Roma: Armando; 2007.
[3] Morin E. I sette saperi necessari all'educazione del futuro. Milano: Raffaello Cortina; 2001.

[4] Wilson T, Holt T, Greenhalgh T. Complexity science: complexity and clinical care. BMJ 2001;323(7314):685-8.

[5] Plsek PE, Wilson T. Complexity, leadership, and management in healthcare organisations. BMJ 2001;323(7315):746-9.

[6] Whittle J, Bosworth H. Studying complexity is complex. J Gen Intern Med 2007;22 Suppl 3:379-81.

[7] Agency for Healthcare Research and Quality (AHRQ). Definition: Complex Patient, Funding Opportunity Announcement (FOA). Technical Assistance Conference Call. October 15, 2007.

[8] Safford MM, Allison JJ, Kiefe Cl. Patient complexity: more than comorbidity. The vector model of complexity. J Gen Intern Med 2007;22 Suppl 3:382-90.

[9] Upshur RE, Tracy S. Chronicity and complexity: is what's good for the diseases always good for the patients? Can Fam Physician 2008;54(12):1655-8.

[10] Nardi R, Scanelli G, Corrao S, lori I, Mathieu G, Cataldi Amatrian R. Co-morbidity does not reflect complexity in internal medicine patients. Eur J Intern Med 2007;18(5):359-68.

[11] Singh H, Petersen LA, Thomas EJ. Understanding diagnostic errors in medicine: a lesson from aviation. Qual Saf Health Care 2006;15(3):159-64.

[12] Fraser SW, Greenhalgh T. Coping with complexity: educating for capability. BMJ 2001;323(7316):799-803.

[13] Martin CM, Sturmberg JP. General practice - chaos, complexity and innovation. Med J Aust 2005;183(2):106-9.

[14] Smith R. Where is the wisdom. . ? BMJ 1991;303(6806):798-9.

[15] Pelletier K. Conventional and integrative medicine: evidence based? Sorting fact from fiction. Focus Alternative Complement Ther 2003;8(1):3-6.

[16] Gill P, Dowell AC, Neal RD, Smith N, Heywood P, Wilson AE. Evidence based general practice: a retrospective study of interventions in one training practice. BMJ 1996;312(7034): 819-21.

[17] Jonas WB. Scientific evidence and medical practice: the "Drunkard's Walk". Arch Intern Med 2009;169(7):649-50.

[18] Cartabellotta A. La medicina basata sulle evidenze: criticità e prospettive. Recenti Prog Med 2006;97(11):640-6.

[19] Pagliani S. Incertezza e variabilità in medicina. http:// www.partecipasalute.it/cms/files/Pagliani-testo-referenze. pdf

[20] Liberati A. Centro Cochrane Italiano, XIII Riunione Annuale Network Cochrane Italiano. Workshop. Napoli, 4 novembre 2008.

[21] American Academy of Family Physicians, American Academy of Pediatrics, American College of Physicians, American Osteopathic Association. Joint Principles of the Patient Centered Medical Home. 2007. http://www.pcpcc.net/node/14

[22] Gambassi G. EBM o Evidence Biased Medicine. Dai trial clinici al mondo reale. $53^{\circ}$ Congresso Nazionale SIGG, XIII Congresso Nazionale FADOI, 2008.

[23] European Medicine Agency. Note for Guidance on Studies in Support of Special Populations: geriatrics. March 1994. CPMPICH: 379-95.

[24] Van Spall HG, Toren A, Kiss A, Fowler RA. Eligibility criteria of randomized controlled trials published in high-impact general medical journals: a systematic sampling review. JAMA 2007;297(11):1233-40.

[25] Pincus T. Analyzing long-term outcomes of clinical care without randomized controlled clinical trials: the consecutive patient questionnaire database. J Mind-Body Health 1997;13(2):3-31.

[26] D’Agostino Jr RB, D'Agostino Sr RB. Estimating treatment effects using observational data. JAMA 2007;297(3):314-6.

[27] Roland M, Torgerson DJ. What are pragmatic trials? BMJ 1998;316(7127):285.

[28] Macpherson H. Pragmatic clinical trials. Complement Ther Med 2004;12(23):136-40. 
[29] Cherubini A. Quali studi clinici controllati?, http://www. sigg.it/diapositive52/congresso/01/04cherubini.pdf

[30] Vandenbroucke JP, Psaty BM. Benefits and risks of drug treatments: how to combine the best evidence on benefits with the best data about adverse effects. JAMA 2008;300(20): 2417-9.

[31] Largo agli studi osservazionali. BIF 2008;XV(6):255-6. Editoriale.

[32] Concato J, Shah N, Horwitz RI. Randomized, controlled trials, observational studies, and the hierarchy of research designs. $\mathrm{N}$ Engl J Med 2000;342(25):1887-92.

[33] Chan KB, Man-Son-Hing M, Molnar FJ, Laupacis A. How well is the clinical importance of study results reported? An assessment of randomized controlled trials. CMAJ 2001;165(9):1197-202.

[34] Jaeschke R, Singer J, Guyatt GH. Measurement of health status. Ascertaining the minimal clinically important difference. Control Clin Trials 1989;10(4):407-15.

[35] Revicki D, Hays RD, Cella D, Sloan J. Recommended methods for determining responsiveness and minimally important differences for patient-reported outcomes. J Clin Epidemiol 2008;61(2): $102-9$.

[36] Tricoci P, Allen JM, Kramer JM, Califf RM, Smith Jr SC. Scientific evidence underlying the ACC/AHA clinical practice guidelines. JAMA 2009;301(8):831-41.

[37] When clinical practice guidelines fail. Lancet 2005; 365(9469): 1440. Editorial.

[38] Grimshaw J, Eccles M, Thomas R, Maclennan G, Ramsay C, Fraser $C$, et al. Toward evidence-based quality improvement. Evidence (and its limitations) of the effectiveness of guideline dissemination and implementation strategies 1966-1998. J Gen Intern Med 2006;21 Suppl 2:S14-20.

[39] Shaneyfelt TM, Centor RM. Reassessment of clinical practice guidelines: go gently into that good night. JAMA 2009;301(8): 868-9.

[40] van Weel C, Schellevis FG. Comorbidity and guidelines: conflicting interests. Lancet 2006;367(9510):550-1.

[41] Tinetti ME, Bogardus Jr ST, Agostini JV. Potential pitfalls of disease-specific guidelines for patients with multiple conditions. N Engl J Med 2004;351(27):2870-4.
[42] Boyd CM, Darer J, Boult C, Fried LP, Boult L, Wu AW. Clinical practice guidelines and quality of care for older patients with multiple comorbid diseases: implications for pay for performance. JAMA 2005;294(6):716-24.

[43] O'Connor PJ. Adding value to evidence-based clinical guidelines. JAMA 2005;294(6):741-3.

[44] Heath I, Rubinstein A, Stange KC, van Driel ML. Quality in primary health care: a multidimensional approach to complexity. BMJ 2009;338:b1242.

[45] Kubler-Ross E. On Death and Dying. Florence, KE: Routledge; 1973.

[46] Cronje RJ, Freeman JR, Williamson OD, Gutsch CJ. Recognizing and managing clinical uncertailnly. Lab Med 2004;35(12): 724-31.

[47] Tsevat J, Dawson NV, Wu AW, Lynn J, Soukup JR, Cook EF, et al. Health values of hospitalized patients 80 years or older. HELP Investigators. Hospitalized Elderly Longitudinal Project. JAMA 1998;279(5):371-5.

[48] Protheroe J, Fahey T, Montgomery AA, Peters TJ. The impact of patients' preferences on the treatment of atrial fibrillation: observational study of patient based decision analysis. BMJ 2000;320(7246):1380-4.

[49] Salkeld G, Cameron ID, Cumming RG, Easter S, Seymour J, Kurrle SE, et al. Quality of life related to fear of falling and hip fracture in older women: a time trade off study. BMJ 2000;320(7231):341-6.

[50] Epstein RM, Alper BS, Quill TE. Communicating evidence for participatory decision making. JAMA 2004;291(19):2359-66.

[51] Gordon DR. Clinical science and clinical expertise: changing boundaries between art and science in medicine. In: Lock MM, Gordon DR (eds). Biomedicine Examined. Boston, MA: Kluwer Academic, 1988:pp. 257-95.

[52] Tonelli MR. The philosophical limits of evidence-based medicine. Acad Med 1998;73(12):1234-40.

[53] Ricci S. L'applicazione dell'evidenza scientifica. Neurol Sci 2004;25:S519-21.

[54] Liberati A. Un decennio di EBM: un bilancio non proprio imparziale. In: Liberati A (ed). Etica, conoscenza e sanità. EBM tra ragione e passione. Roma: II Pensiero Scientifico, 2005. 